\title{
Heavy-metal contamination impacts Au biogeochemical cycling
}

\author{
SK SANYAL ${ }^{1,2}$, J BRUGGER $^{3}$, B ETSCHMANN $^{3}, \mathrm{~F}$ \\ REITH $^{1,2}$, J SHUSTER $^{1,2^{*}}$ \\ ${ }^{1}$ School of Biological Sciences, The University of Adelaide, \\ Adelaide, South Australia 5005, Australia \\ ( ${ }^{*}$ corresponding author: \\ jeremiah.shuster@adelaide.edu.au) \\ ${ }^{2}$ CSIRO Land and Water, Environmental Contaminant \\ Mitigation and Technologies, PMB2, Glen Osmond, \\ South Australia 5064, Australia \\ ${ }^{3}$ Monash University, Clayton, Victoria 3800, Australia
}

Placer gold particles are progressively transformed through (bio)geochemical processes under near-surface weathering environments. Previous studies have focused on $\mathrm{Au}$ biogeochemical cycling from particles obtained from "pristine" environments. The structure and chemistry of gold particles have been used to interpret past biogeochemical processes contributing to particle transformation. Little is known, however, about the influence of heavy-metal contamination on $\mathrm{Au}$ cycling and is therefore worth investigation. In doing so, this study assessed particle structure and chemistry to interpret direct chemical effects of mercury on particle morphology and analysed the genome of bacteria residing on particles to identify possible mechanisms in which heavy metals could indirectly affect particle biotransformation processes. River sediments from the De Kaap Valley (South Africa) contained gold particles and different heavy metals derived from (artisanal) mining activity. Among these heavy metals, mercury is known to chemically interact with gold and alter both gold particle morphology and surface textures. It is possible that mercury "erased" structural evidence (nugget-like morphology or secondary gold structures) of past biogeochemical processes. From a genomic perspective, heavy metals could indirectly affect gold particles by exerting a selective pressure on bacteria residing on particles. For example, Arthrobacter sp. and Pseudomonas sp. are representative microbes that could contribute to particle biotransformation and were isolated from particles (not directly affected by mercury). The genomes of these Au-tolerant isolates contained a diverse range of heavy-metal resistant genes. Particles harbouring Au-tolerant bacteria, with heavy-metal resistant genes, contained three times more pure gold relative to particles with less Au-tolerant bacterial. Therefore, heavy-metal contamination derived from anthopengic activity can have a direct or indirect effect on $\mathrm{Au}$ biogeochemical cycling within natural environments. 